\title{
La Sociología Profesional y su Legítima Identidad en el Campo de las Ciencias Sociales
}

Justino Gómez de Benito ${ }^{1}$

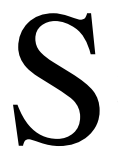
i en alguna época no lejana se consideró ilegítima la dimensión profesional de la sociología y el saber del oficio de sociólogo no se quiso contaminar con las demandas sociales « de la gente », con otros profesionales « de terreno », con el sector privado de la economía o con los requerimientos del mercado, hoy no podemos dejar de reconocer que la transformación de los campos profesionales de los sociólogos, de sus prácticas y de sus competencias, nos están exigiendo, a quienes tenemos responsabilidades de formación de jóvenes sociólogos, buscar los legítimos fundamentos de un saber y de un saber hacer sociológico que se ha resignificado históricamente desde la realidad misma en la que se ha practicado, aplicado y ejercido. La identidad profesional de la sociología construida desde las múltiples modalidades del oficio de sociólogo, se ha ido construyendo como una doble transacción que desde su origen y trayectoria incluye sus propias tensiones internas, su pertenencia a contextos particulares, su relación con disciplinas hermanas o vecinas y su obsesión por dar cuenta de una sociedad que se reconfigura y se resignifica permanentemente.

\section{Una mirada desde la historia de las Ciencias Sociales}

La génesis de las Ciencias Sociales está estrechamente relacionada con la libertad (de individuos y sociedades) y los cambios sociales los inspiraron.

1 Dr. En Sociología, Director del Departamento de Sociología, Universidad Católica Cardenal Raúl Silva Henríquez 
Hablamos de un período (1815-1818) marcado por profundas transformaciones políticas, económicas, sociales y culturales. La sociedad del «Ancien Régime» reposaba sobre los tres órdenes tradicionales: la nobleza, el clero y la realeza. La Revolución Francesa, seguida de las reformas del Primer Imperio dio lugar al estallido de nuevas concepciones de la sociedad en Europa y en el mundo occidental. En Francia, en Alemania y en Austria nacieron y se extinguieron los más diversos regímenes políticos ${ }^{2}$. Algunos pensadores de lo social (S.Simón 1760-1825) y los primeros sociólogos (Comte, Durkheim) partidarios de un orden social estable vieron en esta situación los signos de la debilidad de las sociedades, de una patología del organismo social. Sobre las ruinas del derecho natural, tan querido de los racionalistas del Siglo de las Luces, se articularon las doctrinas más retrógradas que promovían el retorno al antiguo régimen; pero también las más utópicas y audaces.

En este escenario todas las propuestas de los sociólogos intentaban responder a la misma pregunta, ¿cómo poner término a la evidente crisis social que atravesaba Europa?. Las soluciones eran divergentes: la filosofía social de Comte, el socialismo científico de Marx, el moralismo de Durkheim, etc. El desafío era cómo aplicar la nueva ciencia en pro de una reforma social.

Por una parte los intervencionistas consideraban que la sociología debía contribuir a sanar (o al menos a suavizar) los males que sufría la sociedad. El positivismo de S.Simón (la ciencia del hombre o fisiología social) y de Comte (Física social o sociología) ocupan el lugar más extremo: darse como finalidad explícita reformar la sociedad a fin de llevarla hacia el espíritu industrial al espíritu positivo. Para Comte el trabajo del sociólogo es el conocimiento de las leyes de las sociedades, en función de su transformación hacia la sociedad positiva. Para Marx, si los filósofos no habían hecho más que interpretar el mundo y lo que importaba era transformarlo, le cabía al cientista social asumir este compromiso. En todo caso, la preocupación por la práctica de las personas en orden a cambiar sus condiciones sociales de existencia, es una de las claves teóricas del materialismo histórico.

2 Cfr. J.P. DURAND y R. WEIL : « Sociologie Conteporaine »; Ed. Vigot, París, 1989. 


\section{LA SOCIOLOGÍA PROFESIONAL Y SU LEGÍTIMA IDENTIDAD EN EL CAMPO DE LAS CIENCIAS SOCIALES / Gómez de Benito}

Por otra parte los neutralistas (Max Weber y W.Pareto), invocan la imposibilidad que tiene la ciencia para justificar los valores de la acción. En el medio de ambas posturas, E.Durkheim plantea que la ciencia debe ir referida y desembocar en una acción práctica, aunque no sea de inmediato, sino que requiera de un cierto tiempo.

Mi hipótesis es que la historia de la intervención en Ciencias Sociales es la historia misma de la Sociología, de la Psicología, (de la psicosociología) y del Trabajo Social ${ }^{3}$. La historia de las desigualdades sociales y de la marginación es la historia de una ciencia de lo social, de los problemas de la sociedad industrial y los cambios que conlleva. Es la historia también de los intentos por intervenir y superar estos problemas desde el conocimiento que aporta este saber científico.

El supuesto básico de toda intervención social es la conceptualización del hombre como ser social y responsable de su propio destino. En este sentido corresponde con una idea absolutamente moderna del hombre y de su quehacer en el mundo. Un quehacer que puede preverse, planificarse, programarse y ejecutarse, y que pretende no dejar nada en manos del azar. Podríamos relacionar esta comprensión con la referencia a la acción racional con arreglo a fines, de M. Weber y su comprensión del proceso de racionalización e intelectualización ${ }^{4}$

Una lectura profunda de la realidad histórica nos descubre al menos tres etapas en el proceso de comprensión y construcción teórica de la intervención. Desde comienzos del S. XX hasta 1920 se comprende la intervención como caridad y voluntariado, predominantemente, aunque se inicia un proceso de profesionalización. Entre los años 20 y 40 se inicia el desarrollo de una teoría con fundamentos en las fuentes norteamericanas. Con posterioridad a estas fechas se comienza a dejar de lado la teoría americana y se va introduciendo progresivamente una intervención en términos de tareas. En esta última fase, la autora citada, hace notar la valiosa contribución que la historia de la intervención latinoamericana ha aportado a la redefinición del Trabajo Social ${ }^{5}$.

3 Cfr. GORRI GOÑI, A.: "La intervención psicológica en Política Social y Servicios Sociales”, Ed. Libertarias/ Prodhufi, Navarra, 1995

4 Cfr: M.Weber: "El científico y el político"; Alianza Editorial, Madrid, 1979

5

(Idem, pp 28-35) 
Las teorías de las Ciencias Sociales que históricamente explican los distintos tipos de Intervención social son, según Cristina de Robertis ${ }^{6}$, el funcionalismo, el estructuralismo, el culturalismo e interaccionismo simbólico, la teoría de sistemas y el marxismo. Estas teorías entregan las claves explicativas a los modos de intervención. Una comprensión de la intervención social está fundamentada en alguna teorización sobre la acción social. Este supuesto no implica necesariamente su contrario. Sin embargo, sí sostenemos, que la comprensión de lo social como "cuestión social”, como problema social y de la acción sobre lo social (intervención) está mediatizado por los diferentes contextos sociopolíticos y económicos. Estos contextos o escenarios han sido parte de las formulaciones y reformulaciones de los capitalismos y socialismos reales, en sus diferentes versiones. Las diferentes versiones del capitalismo, desde el siglo XIX a la fecha ha implicado una comprensión de la realidad social expresada como "cuestión social" ", una comprensión también del trabajo y del rol del Estado.

Sintetizando las diferentes teorías de las ciencias sociales se puede afirmar que además de aportar los elementos para el análisis de los procesos sociales, permiten comprender las situaciones específicas de los sujetos, sean individuos, grupos o comunidades. Obviamente, estos elementos de comprensión de la situación son los que determinan la orientación de la Intervención.

\section{A propósito de la identidad profesional de la sociología}

Abordar el tema de la comprensión teórica del ejercicio profesional del sociólogo implica preguntarse por las dinámicas profesionales que la atraviesan desde sus orígenes: que factores-fuerzas han impulsado en una dirección o en otra. La preocupación por una comprensión científica de lo social a través de un trabajo en y sobre lo social implica necesariamente abordar el proceso que ha seguido el ejercicio práctico de la sociología, de su profesionalización, de sus problemas de legitimación y sus desafíos para el futuro. Este ejercicio intenta contribuir a la sociolo-

6 DE ROBERTIS, C.: "Metodología de la Intervención en Trabajo Social”, Ateneo, Barcelona, 1992

7 CASTEL, R.: "Les métamorphoses de la question sociale”, PUF, París, 1996 


\section{LA SOCIOLOGÍA PROFESIONAL Y SU LEGÍTIMA IDENTIDAD EN EL CAMPO DE LAS CIENCIAS SOCIALES / Gómez de Benito}

gía de la sociología y a la sociología del conocimiento. La comprensión de los procesos de construcción de las identidades de la sociología en el transcurso de su historia y los aportes recíprocos entre práctica disciplinar y práctica profesional de los sociólogos al saber y al saber hacer de la sociología son hoy todavía caminos poco explorados. Estudiar este tema permitirá aclarar la diversidad de las nuevas demandas sociales a partir de las cuales se estructuran nuevas formas de identidad profesional.

Los aportes teóricos de C. Dubar ${ }^{8}$ nos permiten transferir sus conceptos a nuestra propia disciplina: La identidad de la sociología se ha ido construyendo como una doble transacción. Una transacción biográfica referida a la comprensión de su propia trayectoria y tensiones internas y una transacción relacional en relación a contextos particulares y disciplinas hermanas o vecinas. Esta preocupación no es nueva. A comienzos del S. XX Durkheim y Weber quisieron delimitar un campo autónomo para la sociología. La lucha por la legitimidad entonces se daba en el espacio de la Universidad. La sociología de estos clásicos se situó en la Universidad, y se desarrolló como una sociología académica.

Hoy la sociología también está cruzada por tensiones de legitimidad, pero en otras direcciones que las de antaño. Por una parte ha habido desde mediados de siglo una expansión (con grandes diferencias según continentes y países) de la oferta universitaria de la sociología. Por otra, las transformaciones del rol del Estado, los nuevos problemas y desafíos que plantean las sociedades en sus procesos de desarrollo, el resurgir de situaciones de exclusión o de acrecentamiento de las desigualdades sociales, han provocado demandas sociales de las más variadas formas que se van constituyendo en posibilidades nuevas de aplicación de un saber sociológico que ofrezca no sólo nuevos conocimientos sino también desarrollar competencias fiables en el plano de la acción, alternativas de solución y caminos de construcción de futuros deseables.

Pero el campo de la acción profesional de la sociología se ha ido progresivamente desarrollando tanto desde los poderes y servicios del Estado (cuando era Estado-providencia), como desde los espacios universitarios,

8 DUBAR, C.: «La socialisation: construction des identités sociales et professionneles », Armand Colin, París, 1991 
centros de investigación y equipos de consultoría y servicios profesionales. Este desarrollo, a juicio de algunos en forma anárquica, de formas profesionales de intervención social, que va desde los trabajadores sociales clásicos a los ingenieros sociales, pasando por los expertos y los consultores, corre el riesgo de crear una confusión peligrosa entre lo social y lo sociológico, entre acción social y trabajo sociológico.

Es evidente que el campo de la Sociología se ha abierto y se ha diversifica$\mathrm{do}^{9}$. La mayor parte de los sociólogos que hoy se titulan no pueden ejercer funciones de docencia o investigación en universidades o Instituciones de investigación de alto nivel como era el sueño de las generaciones de comienzos y mediados de siglo. La masificación de las ofertas de acceso a la licenciatura en Sociología y de la obtención del título profesional de sociólogo está situando a jóvenes sociólogos en una pluralidad de lugares y situaciones, roles, tareas, funciones y servicios , que ejerce una presión de dinamización y diversificación del campo de la sociología profesional. Este ejercicio profesional del sociólogo, habitualmente puede hacerse sinónimo de intervención social ${ }^{10}$. Además de la ampliación del campo de trabajo se ha diversificado también el modo de hacer sociología, que además del ámbito clásico de la investigación, ha ido incluyendo el de los estudios de problemas focalizados, la planificación, la intervención y la gestión.

Esta reflexión, expresión de una búsqueda que como equipo de investigación estamos haciendo en nuestra universidad, quiere comprender este proceso de transformación del campo profesional de la sociología, de sus identidades, sus prácticas, sus comprensiones y sus competencias ${ }^{11}$. El hilo conductor de la investigación es el de la intervención. La multiplicidad de prácticas de intervención por los profesionales de la sociología

9 Cfr. «Travaux sociologiques » $N^{\circ} 3$, LSCI, 1987; Tambièn el $N^{\circ}$ especial de la Revue d l'Institut de Sociologie la Universidad Libre de Bruselas en 1988. En 1992 la Revue Suisse de Sociologie dedicó un $\mathrm{n}^{\circ}$ a la sociologìa de Suiza y a la sociología en Suiza. En 1993 se realizó en Lieja (Bèlgica) un coloquio sobre el tema, organizado por l'Association internationale se sociologie de Langue Française (AISLF).

10 ACSALF: Colloque 1981 «L'intervenction sociale »; Editions Coopératives Albert Saint-Martin, Quebec, 1982

11 Cfr. GOMEZ J., SANDOVAL M., SANCHEZ, D., y RIVERA M.: «Intervenir lo social: nuevas prácticas, competencias e identidades para los profesionales de las Ciencias Sociales (Sociología, Trabajo Social y Psicología). Informe de investigación ; Universidad Católica Cardenal Raúl Silva Henríquez ; 


\section{LA SOCIOLOGÍA PROFESIONAL Y SU LEGÍTIMA IDENTIDAD EN EL CAMPO DE LAS CIENCIAS SOCIALES / Gómez de Benito}

permite afirmar que después de un siglo de desarrollo de la sociología, «el terreno » y la Universidad, la teoría y la práctica, el análisis y la intervención forman parte de la construcción de nuevas identidades de los sociólogos y por ello de la propia sociología (ciencia aplicada, disciplina y profesión). Nuestra posición es que la identidad de la sociología se construye de hecho desde la pluralidad y la interacción de las múltiples formas de ejercicio y de aplicación del saber sociológico que es disciplinar y profesional. Atomización sin comunicación conduce a la desintegración y pérdida de identidad.

Si los marcos institucionales de legitimación de los sociólogos están situados en los espacios universitarios, las competencias profesionales que resultan de las prácticas de intervención social, en todo su espectro y rápida evolución, han creado una dinámica nueva en la sociología.

El desafío es conocer cómo desde las nuevas prácticas los sociólogos experimentan la pertenencia a una disciplina que les aporta un saber del que ellos se sirven para su práctica de intervención social. Una profesión debe encontrar los medios para aportar competencias operativas ante las demandas sociales o del mercado laboral. Pero también las competencias profesionales y las competencias científicas (teóricas y metodológicas) requieren de reciprocidad y afirmación común.

Es también nuestro interés contribuir con este trabajo a aclarar el problema de la construcción y transformación de las identidades profesiones de la sociología, desde las nuevas demandas que se han ido presentando y a partir de las cuales también se han ido estructurando nuevas formas de aplicación de nuestro saber sociológico.

Preguntarse por la trayectoria de la dimensión de intervención en la práctica profesional de los sociólogos implica situar el problema en la identidad de la propia sociología. ¿Qué es la sociología, qué ha sido y qué va a ser en el futuro?, ¿Quiénes y dónde han hecho sociología?, ¿Qué tiene que ver la sociología con la intervención en la realidad social?. Preguntarnos por lo qué es, puede implicar decir lo que hasta ahora ha sido y lo que es hoy. ¿La sociología ha sido más disciplina que profesión?, ¿ha estado más centrada en la investigación fundamental que en la investigación aplicada? 
Pero además es imprescindible cuestionarse como orientación de la investigación, sobre cuáles son los fundamentos de legitimación que ha tenido la profesionalización de la sociología.Y sobre esta pregunta se incluye la siguiente sobre los fundamentos de legitimación de las prácticas profesionales de intervención de la realidad en vistas a su transformación a fin de procurar una situación mejor que la anterior, juzgada así por los responsables de la sociedad, por los investigadores o por los propios sujetos interesados.

Las prácticas de intervención guardan relación con un conjunto de características de un modo de hacer sociología:

1) Una dimensión más profesionalizante (fuera de la Universidad) en vez de una dimensión más disciplinar (más presente en el espacio universitario. Esta práctica ha estado más centrada en la acción por el cambio que en la producción de conocimiento sobre él (analítica).

2) Una sociología interesada más en la investigación empírica que en la investigación fundamental. Esta investigación ha tenido relación con la acción y el cambio, comprometido con él. Aunque investigación empírica no responde a una concepción de ciencia positivista.

3) Un trabajo de producción de conocimiento de la realidad social desde una posición de la ciencia comprometida con valores, y directamente con un modo de construir la sociedad (humanismo, democracia, socialismo, libertad, etc.) versus un tipo de ciencia neutra, al servicio (hipotéticamente) de sí misma.

4) Otras muchas tensiones están presentes en la dinámica del campo de ejercicio disciplinar y profesional de la sociología que consideramos importante destacar: teoría sociológica y metodología; objetividad y/o subjetividad en la investigación; implicación o lejanía del sociólogo respecto al objeto de estudio; investigación disciplinar o inter-pluri o trans disciplinar; investigación al servicio del bien público o en beneficio del sector privado, producción de conocimiento versus su aplicación y la gestión de su aplicación, etc. 


\section{LA SOCIOLOGÍA PROFESIONAL Y SU LEGÍTIMA IDENTIDAD EN EL CAMPO DE LAS CIENCIAS SOCIALES / Gómez de Benito}

Estas tensiones muestran la pluralidad de manifestaciones como se expresan hoy las prácticas de la sociología: desde una expresión más disciplinar, pasando por múltiples formas y niveles del ejercicio profesional, para llegar al terreno de la complejidad de los saberes y competencias que se le demandan. Producir conocimiento sobre esta diversidad de componentes y expresiones de la identidad profesional de los sociólogos nos parece un significativa contribución a la comprensión del « oficio » de sociólogo y al esclarecimiento de la relación entre las dimensiones disciplinar y profesional de la sociología.

Nuestra hipótesis es que existe una identidad compartida por la comunidad de sociólogos. Ahora bien esta identidad está fragmentada y tensionada por todas las comprensiones que la propia sociología tiene de lo social como realidad y de las expectativas o de los estereotipos que los actores sociales, políticos y económicos se hacen de nuestro saber y de nuestras competencias.

\section{La construcción de una identidad legítima}

La necesidad de legitimar la sociología como ciencia y de definir sus límites como disciplina autónoma, hizo que todo el esfuerzo de Durkheim y de Weber en Europa se dirigiera hacia situar a la Sociología entre las demás ciencias humanas y junto a las ciencias naturales, sobrevalorando la ubicación de un saber científico dentro de la Universidad. Esta sociología académica, formaba parte de una cultura general y humanista, y estaba preocupada de construir la disciplina como ciencia capaz de transmitir teorías, métodos y resultados para afirmar su autonomía al lado de las otras ciencias humanas anteriores a ella, como la economía, la historia, el derecho, la psicología y la etnología.

Sin embargo, este esfuerzo por legitimar la disciplina en el espacio universitario no se quedó reducido a la construcción de teorías y ensayos. Los sociólogos supieron responder a las demandas y desafíos de una época necesitada de saberes centrados en las condiciones de nacimiento y desarrollo de una sociedad industrial y republicana. Para F. Jonas ${ }^{12}$, 
el origen y el sentido de la creación de una ciencia sociológica; a la vez empírica y racional, acumulativa y objetiva, ha tenido como tarea resolver dos problemas de la sociedad moderna:

1) El de la emancipación de las sociedades. Apoyándose en Durkheim y Weber, Jonas sostiene que las sociedades occidentales han estado guiadas por la voluntad de emancipación, de liberación en relación con los prejuicios, a las tradiciones, a los fanatismos, de emancipación de la razón lúcida y de la voluntad moral ilustrada. La emancipación es entendida como la toma de conciencia por el hombre de la autonomía de la razón en la vida práctica y en el conocimiento. Es el gran tema kantiano.

2) La integración de la sociedad. Para Jonas, su integración es lo que hace que una sociedad persista, que sea coherente y al menos que conserve una coherencia suficiente como para perdurar. La primera misión de una teoría sociológica es explicar la integración, sostiene. Muchas soluciones son posibles: la naturalista que confía en que el hombre es un ser social por su naturaleza. En este caso la integración se debería explicar fácilmente ya que la condición fundamental está resuelta por la naturaleza sociológica original del hombre.

Pero si el hombre es ser social por naturaleza, ¿por qué tantos conflictos, guerras, revoluciones, miseria en una era de prosperidad?. Y si el hombre es fundamentalmente individualista, ¿cómo se comprenden los avatares de la historia, las violencias, las guerras, etc.? Los sociólogos individualistas están obligados a explicar la integración a partir de una naturaleza humana hecha para rechazarla. En este caso, ¿dónde se sitúa el fundamento de la vida social?. Las luchas y las guerras no son siempre constantes. Las sociedades perduran por siglos y milenios. ¿Dónde está el fundamento de los vínculos sociales?

Se trata de un contrato, según Rousseau. Se trata de la religión, el derecho divino, según Bossuet y los sociólogos cristianos. Otras fórmulas son también posibles y han estado presentes a lo largo de la historia de la sociología. Uno de los méritos de la historia de la Sociología de Jonas es que la explicación de la integración se mantiene como centro de la 


\section{LA SOCIOLOGÍA PROFESIONAL Y SU LEGÍTIMA IDENTIDAD EN EL CAMPO DE LAS CIENCIAS SOCIALES / Gómez de Benito}

investigación, por el camino de la elaboración de una ciencia experimental y racional auténtica.

Comte había llamado a su sistema de estudio de la sociedad «física social ». El concepto de « sociología » lo introduce para diferenciarse de Quetelet, que también había intentado introducir una física social en 1835 ( «Sur l'homme et le développement de ses facultés ou Essai de physique sociale »). Quetelet, se toma en serio el programa científico del positivismo, consistente en registrar las series de hechos como tales, como lo hacen las ciencias naturales. Los hechos no necesitan la teoría para su interpretación, como planteaba Comte. La teoría se reemplaza por la simple constatación de hechos. La constatación de los hechos sociales y de regularidades que se manifiestan en su sucesión debe poner fin a todas las especulaciones sobre la autonomía de las sociedades humanas. Nunca antes se había intentado explicar la integración de la sociedad a partir de los hechos, solamente. Cuando Quetelet muestra que las estadísticas han sido capaces de descubrir regularidades, manifiestamente independientes de los valores y las instituciones, ha podido describir la cohesión social, más allá de las instituciones y los valores. Los hechos deben hablar por sí mismos.

Independientemente de la cuestión de saber si las teorías sociológicas son justas o falsas, la observación de las realidades sociales concretas gana importancia en la medida que los sistemas sociales se transforman. El conocimiento sobre el presente ya no puede ser garantizado por la tradición. Solamente la investigación puede asegurar el verdadero conocimiento. La investigación empírica ha encontrado su legitimidad en tanto ciencia auxiliar de la administración y la importancia de esta ciencia auxiliar se afirma cada vez más en la medida que las transformaciones sociales se han ido acelerando.

El caso de Prusia e Inglaterra son dos casos típicos de la influencia de los cambios sociales en la investigación social. En Inglaterra, país en que el desarrollo industrial tuvo sus primeras manifestaciones, tuvieron también lugar las primeras investigaciones parlamentarias sobre la situación social desde el S. XVIII. Numerosos informes oficiales existían en esa época sobre cada uno de los aspectos de la vida social, hechos por 
especialistas y estadísticos. Marx utilizó mucho estos datos en El Capital. Pero además de estas investigaciones oficiales hay muchas monografías de gran valor. Esta tradición continuó durante el comienzo del S. XIX. En Francia, en cambio hasta mediados de siglo no aparecen estudios empíricos (Villermé, 1840), Buret (1840). Sin embargo, estos trabajos no justificaron la ambición de Quetelet de realizar con la ayuda de la estadística una física social, y remplazar así la teoría sociológica ${ }^{13}$.

El modo de hacer ciencia social para Le Play era a través del contacto con la gente, en terreno, encuestando y observando ${ }^{14}$. El método para él debía interpretarse como una ciencia auxiliar administrativa, no teórica ("las reformas sociales"). Se deja de lado la idea de revolución para interesarse en las realidades de la convivencia humana. Los gobiernos que se apoyaron sobre las investigaciones empíricas pudieron lograr verdaderos progresos, en vez de progresos ideológicos. La investigación empírica atacó de frente a la teoría especulativa considerada como culpable de intenciones revolucionarias.

En Alemania la investigación empírica aplicada después de la $2^{\mathrm{a}}$. mitad del S. XIX fue heredera del interés de Dilthey « de comprender el universo espiritual y social a partir de lo vivido puramente empírico ». Sin embargo, salvo por Weber, la investigación empírica estuvo separada de la teoría. El conocimiento científico para él concierne la realidad empírica y las posibilidades de acción técnica. El conflicto de Max Weber con Alfred Weber, Herkner y Smoller era la diferencia de intenciones. Para ellos la investigación tenía una intención política y social, a fin de mejorar la legislación en vigor, o facilitar la fundamentación de nuevas leyes. Para Weber la investigación tiene una finalidad puramente científica. La sociología en tanto ciencia empírica concierne para él una realidad histórica particular, por ende, debe aclarar la significación cultural de los hechos sociales. Así es como Max Weber se distingue de aquellos que querían practicar una investigación empírica sin teoría y de aquellos que se interesaban en la sociología « pura »; (Fue criticado por Parsons por no desarrollar una teoría general). 


\section{LA SOCIOLOGÍA PROFESIONAL Y SU LEGÍTIMA IDENTIDAD EN EL CAMPO DE LAS CIENCIAS SOCIALES / Gómez de Benito}

La rentabilidad operacional del saber, el cálculo de suertes en el campo de la acción reposan sobre esta desmitificación. La distancia crítica y la separación entre la ciencia y la política que Max Weber ha postulado como fundamento de la metodología, son abandonados. La síntesis empírica y la certeza absoluta fundada sobre una concepción del mundo son el nuevo credo del conocimiento científico.

Para Karl Manheim el deber de la sociología es realizar una síntesis en que los métodos empíricos de la investigación sociológica pueden servir para aportar un fundamento a los desarrollos constructivos, y no pueden constituir un fin en sí mismos. Estas síntesis son concebidas como culturales o como la expresión de una orientación política particular. Para Schelting el verdadero conocimiento consiste en «captar la verdadera estructura interna global de la vida histórica». Son estos puntos de vista los que han predominado en la sociología de los años 20 y 30 en Europa. El positivismo estaba siendo superado por la Fenomenología.

La significación no es más, como lo pensaba Weber, una significación subjetivamente pensada o subjetivamente querida, sino también una significación objetivamente dada u objetivamente querida. M. Weber, dice Shutz no ha llegado en sus análisis a esta profundidad. Y es esta profundidad la que se busca explorar, y es esta la tarea del sociólogo. No es la comprensión racional sobre la base de tipos ideales, sino la verdad mediatizada por experiencias vividas o de situaciones particulares lo que es ahora el objeto de la ciencia

Como quería Weber, se va fortaleciendo a lo largo del S. XX una sociología lejos de la separación entre teoría y práctica. Aparece una voluntad apasionada de ejercer una influencia sobre la práctica. La voluntad de descubrir la verdad científica, fundada sobre la reflexión crítica es remplazada por la voluntad de ejercer una acción personal, fundada sobre la creencia en una verdad. «La voluntad de actuar a nivel de la práctica es la condición del conocimiento sociológico» declara Freyer en 1930. G. Lukas plantea que «sólo el que está llamado a realizar el futuro y que tiene la voluntad puede comprender la verdad concreta del tiempo presente ${ }^{15}$. Para Neurath la sociología «es la ciencia de los

15 Citado por Jonas (Op. cit., pág. 387) 
hombres de Estado y de los organizadores, es decir, de los técnicos de la vida social». Oppenheimer y Plenge declaran que ellos ofrecen una concepción del mundo útil a fines prácticos. Para Tönnies la sociología aplicada es la ciencia del hombre de Estado. Su fin es una política inspirada por la ciencia, como lo quería Comte. Sus categorías de comunidad y sociedad ejercieron más influencia como expresión de una voluntad de práctica política que como instrumentos de análisis teórico. Dividió el conocimiento en tres partes: la investigación empírica, la teoría pura y la sociología aplicada.

Para Manheim la «motivación secreta de la sociología es la cuestión de cómo voy a satisfacer tal o cual necesidad» ${ }^{16}$ No se trata del mismo interés de las ciencias de la naturaleza (el conocimiento), sino de necesidades concretas, y por ello en las luchas de los partidos por la primacía de tales o cuales exigencias. Este principio de la parcialidad es radicalizado en el principio de la vinculación al ser de todo conocimiento en el campo de las ciencias sociales.

Para estos autores no es el trabajo crítico del sabio el que aporta la verdad, sino que la verdad es la que se ofrece a aquel que se compromete en las luchas de los partidos de su época y que por ello enriquece su conocimiento del ser. La escala de medida entre lo verdadero y lo falso no es más la crítica científica, sino la victoria en la lucha de los partidos, a propósito de «la interpretación del ser», una expresión que antes que de Mannheim venía de Heidegger.

Dahrendorf habla de una culpabilidad de Weber debido a que nunca reconoció la responsabilidad moral de la sociología ante el humanismo por su insistente libertad sobre los juicios de valor. En este sentido König visualiza la sociología fundamentalmente como una investigación metodológicamente empírica, plantea los valores de un humanismo activo y realista como la condición primordial de su trabajo. Toma la posición de Tönnies. «la sociedad podrá ser un día lo que nosotros querramos que sea; sólo debemos decidir quererlo de verdad. Otros representantes de esta sociología comprometida son T. Adorno y T. Geiger. Para Adorno « lo real no se entrega más que a la mirada que lo contempla bajo el ángulo

16 Citado por Jonas (Op. cit. pág. 390) 


\section{LA SOCIOLOGÍA PROFESIONAL Y SU LEGÍTIMA IDENTIDAD EN EL CAMPO DE LAS CIENCIAS SOCIALES / Gómez de Benito}

de un interés real, bajo el de una sociedad libre, de un Estado justo, de la emancipación del hombre.

La teoría debe desembocar en la práctica, la sociedad debe asumirse, la racionalidad de los medios debe dar paso a la racionalidad de fines. Esta convicción presupone el rechazo a la validez de las ciencias positivas en general. Para quien mira las cosas desde el punto de vista de la redención, es decir, desde el fin de la historia, la ciencia no es el medio del conocimiento (que le es dado fuera de él), es un instrumento en vistas al cambio.

\section{El oficio de sociólogo, entre la cátedra y el terreno:}

(La experiencia de la Francia de postguerra)

La sociología francesa contemporánea ${ }^{17}$ es impensable sin el aporte de la sociología comprensiva alemana y de los diversos paradigmas americanos. En este sentido es parte de una historia singular y de una historia colectiva. Singular por la fortaleza de su institucionalización, pero a la vez por lo precario de su desarrollo. Hubo que esperar al período de postguerra para hablar verdaderamente de desarrollo. Es también una historia singular en tensión permanente con el campo político, tensionado entre el compromiso y la protesta militante, por un lado y el «servicio al príncipe» a través de las peticiones de investigación, del otro.

Después del esfuerzo de Durkheim de dejar una herencia unitaria de la sociología situada en la Universidad, la experiencia francesa de desarrollo de la sociología de la postguerra está marcada por dos contextos que han contribuido a conformar prácticas sociológicas diferentes: el contexto de crecimiento (rol protagónico del Estado) hasta la década de los '70 y el de la crisis y reconstrucción neoliberal de la economía, en las décadas posteriores.

Aparece en los años de postguerra una nueva generación de jóvenes sociólogos que recoge la tendencia predominante en Estados Unidos:

17 JEAN MICHEL BERTHELOT (sous la direction) : «La sociologie française contemporaine », PUF Fondamental, París, 2000 
la investigación empírica y cuantitativa. Surge en este período el sociólogo investigador (académico y profesional, de cátedra y de terreno). El ejemplo de los sociólogos franceses es emblemático: R. Boudon, P. Bourdieu, M. Crozier, H. Mendras, A. Touraine y E. Morin, como figuras más destacadas, hasta hoy. Detrás de ellos viene otra generación: Sainsaulieu, Dubet, Dubar, Maffesoli, De Gaulejac y otros más que sería largo enumerar.

Apenas terminada la Guerra, Gurvitch volvió de Estados Unidos, donde se había refugiado, y convenció al C.N.R.S. de crear el Centre d'études sociologiques (C.E.S.). Del otro lado del Atlántico traía la idea de que la sociología debía desarrollarse en Francia por la investigación de terreno. Estaba convencido de que la sociología debía ser « hiperempírica y superrelativista ». Friedmann, comunista como Gurvitch, pero más sociólogo que partidario, estudió la realidad de las fábricas de Stalingrado, pero después estudió las de Detroit. Este ejemplo impulsó a Touraine, joven sociólogo entonces, a dejar su familia e irse a trabajar a las minas del Norte, e incluso llegar hasta Chile y bajar a las profundidades de Lota. Friedmann fue el verdadero mentor de la generación de sociólogos de la postguerra en Francia. Cuenta H. Mendras: « ayudando a cada uno de nosotros a descubrirnos a nosotros mismos, a situarnos en el mundo de la época; convenciéndonos que cambiaríamos el destino nos impulsó a ir a Estados Unidos $»^{18}$

Otro maestro de la época fue Gabriel Le Brass, quien elaboró un método de análisis de la religión cristiana cuyos resultados facilitaron la utilización de sociología al interior de la Iglesia, hasta transformarse en uno de los más grandes demandantes institucionales de investigaciones sociológicas. "Preocupémonos bien de asegurar los hechos, antes de interesarnos en las causas. Así evitaremos el ridículo de encontrar causas sin hechos »: era su principio metodológico.

Stoetzel conoció a Gallup en Estados Unidos que le convenció de introducir en Francia los sondeos de opinión, a pesar de la actitud contraria de R. Aron, quien de manera amigable le expresaba un desprecio profundo por esta práctica: «mon vieux, c 'est idiot ton truc » ${ }^{19}$ Fue el Director

18 H; MENDRAS: «Comment dévenir Sociologue , Actes du Sud, Arles, 1995) 19 «Mi viejo, tu truco es inútil» 


\section{LA SOCIOLOGÍA PROFESIONAL Y SU LEGÍTIMA IDENTIDAD EN EL CAMPO DE LAS CIENCIAS SOCIALES / Gómez de Benito}

del segundo Centre d'etudes sociologiques en 1956. Los acontecimientos de Mayo 68 le superaron. El los criticaba de «sociolalia », perversión de la disciplina.

Estos cuatro hombres hicieron posible la recuperación de la sociología en Francia, pero aún más, le otorgaron un rasgo de identidad propio: la investigación de lo que estaba pasando en Francia. Fue una sociología empírica. Sin embargo, la Universidad no formaba en esta línea. La mayor parte de los estudiantes iban a terreno sin la más mínima experiencia de ubicación.

¿Pero por qué querían ser sociólogos?. Cada uno tenía sus razones, sin embargo, para hacerse sociólogo "es preciso tener una cierta insatisfacción ante la sociedad, y una inquietud sobre su posición personal en ella" ${ }^{20}$. Jóvenes y menos jóvenes, toda la generación de sociólogos en esos años estaba convencida de que su deber era hacer surgir a Francia de la ruina. La injusticia social era difícil de soportar. Había preguntas sin respuesta: ¿por qué la diferencia entre la vida de los palacios y las de los campesinos? Los sociólogos de esos años eran burgueses escandalizados de la realidad francesa, de sus contrastes, de la miseria obrera y de las ruinas del conflicto bélico.

Cada uno de estos sociólogos de postguerra había hecho una gran opción por la sociología. Era una opción política, pero por la política en su sentido más noble, como servicio público, servicio al Estado; sea como funcionario o como hombre político. Las ciencias sociales eran para esa generación una forma concreta de servicio público, en un sentido de servir al bien de todos y de la ciudad. Raymond Aron decía que hay tres tipos de sociólogos: el consejero de palacio, el médico del pueblo y el gran sacerdote de la ciencia. Para la mayor parte de esta nueva generación el mayor interés estaba en ser «médico del pueblo », de un país que salía de las ruinas. Algunos se tentaban con la invitación que se hacía de dar « un año de la vida por Europa ». Calmar la miseria del pueblo y construir una ciudad más justa, eran las preocupaciones y las ambiciones de muchos de estos jóvenes sociólogos de postguerra. Las posturas políticas eran diferentes, desde los troskistas, estalinistas,

20 Cfr. H. MENDRAS: (Op. cit.). 
socialistas, idealistas o socialcristianos, todos coincidían en un interés por dar repuesta a la cuestión social. Pero las formas también variaban: Unos entraban a la política, otros a las universidades, pero todos desde la investigación empírica ${ }^{21}$.

Aunque no todos fueron a Estados Unidos, esta generación de sociólogos de la guerra fría, aprendieron a hacer sociología a la americana, incluso entre los comunistas, que influenciados por Gurvitch hablaban de la « cuantofrenia americana ». Pero eran un poco marginales en el mundo intelectual y culto de París. Sin embargo en Chicago, Mendras encontró otra realidad: la preocupación por « ir y ver», de sumergirse en el barro de lo social. En Estados Unidos Durkheim era considerado el fundador de toda la ciencia social empírica y rigurosa, mientras en París se le consideraba más bien un filósofo. Este aprendizaje americano se transformó en un rasgo de la sociología de postguerra, de su originalidad y de su extraordinario éxito, precisamente porque la disciplina era nueva y respondía a una demanda de la sociedad. Más que pedir a los campesinos que respondieran cuestionarios, los sociólogos fueron a terreno, a estar y a observar su vida con ellos, conocer la estructura de los pueblos y su relación con el progreso. Lo que Banfield, Hughes y Warner hacían en Chicago se podía hacer en Francia. Unos años más tarde Hughes fue redescubierto por los sociólogos de tradición crítica.

En el C.E.S. se constituían los equipos bajo la orientación de Friedmann: Touraine los obreros, Crozier la administración, Fdofny las poblaciones, Treanton la ciudad, Reynaud los sindicatos, Viviane Isambert la escuela, Madeleine Guilbert las mujeres, Roland Barthes la literatura, Edgard Morin el cine y Mendras los campesinos. H. Le Bras tenía su campo religioso bien delimitado con Desroche, E. Poulat, Fr. Isambert. Por su parte G. Balandier y Paul Mercier fundaban la sociología africana. Gurvith iniciaba la sociología del conocimiento con Duvigneaud y Cazeneuve. Robert Pagés inventaba la psicosociología experimental. Naville se movía entre la psicología y la sociología del trabajo. ${ }^{22}$

21 Cfr. H. MENDRAS: (Op. cit.)

22 LE BRAS, G, ARON, R., GURVITCH, ISAMBERT,F, FRIEDMAN, G. y Otros: « Aspects de la sociologie Française »; Les Editions ouvrières, París, 1967 


\section{LA SOCIOLOGÍA PROFESIONAL Y SU LEGÍTIMA IDENTIDAD EN EL CAMPO DE LAS CIENCIAS SOCIALES / Gómez de Benito}

En una voluntad de fortalecer la democracia en Europa y de ayudar a fortalecerla más allá de sus fronteras, los americanos favorecieron el desarrollo de las ciencias sociales, otorgándoles el poder de hacer reflexionar a la gente y hacerla más inteligente, y con ello mejores demócratas. Con ese fin apoyaron el Departamento de Ciencias Sociales de la Unesco, desde donde se repartió la sociología a todo el mundo. Touraine, Reynaud y Brams fueron enviados a Chile para crear FLACSO, cuya presidencia fue confiada a Friedmann. Otros fueron a Grecia, Irán, Túnez, Togo, convencidos de que la ayuda al Tercer Mundo era provechosa tanto para los expertos como para los estudiantes con los que se compartía el saber.

La mejor manera de caracterizar esta época es decir que esa generación de sociólogos era como una banda de amigos, cada uno decidido a hacer sus trabajos, pero compartiendo las convicciones de que "estábamos inventando una nueva ciencia y una nueva actitud ante la sociedad y los problemas sociales". Cada uno se situaba en su campo de investigación con su equipo dando a conocer lo que investigaba. Nada se dejaba de hacer para lograr una institucionalización fuera de la universidad. Este grupo se sentía como pioneros descubriendo tierras intelectuales nuevas, con instrumentales nuevos. « No sabíamos hacia donde íbamos, pero sabíamos como queríamos ir. Además teníamos la voluntad de tener un papel y una influencia importante en el resurgir de nuestro país », indica $\mathrm{H}$; Mendras ${ }^{23}$.

Después de los acontecimientos de Mayo 68, las tensiones entre sociólogos se acrecentaron en torno al tema del reconocimiento de la sociología crítica y la sociología aplicada y profesionalizante. La posición más puritana defendía en la comisión de sociología del CNRS la tesis de que la sociología debía rechazar toda tentativa de investigación por encargo, orientada por contratos públicos o peticiones privadas que desviaran a la sociología, en tanto ciencia, de sus objetivos primeros.

Por otra parte, un grupo de sociólogos entre los que destaca M. Crozier, J.D. Reynaud, R. Boudon y F. Boudrillard crearon centros de investigación y

23 MENDRAS, H. (Op.cit.) 
acción profesional en las que formaban a los jóvenes sociólogos en procesos de intervención. ${ }^{24}$ Este período, rico en desarrollo disciplinar fue también pródigo en tensiones y malentendidos. Además de las tensiones entre campos de especialización y sobreespecialización, la práctica de la sociología para el común de los sociólogos estuvo marcada por dos referentes difíciles de compatibilizar: el poder político y económico (público y privado), por un lado, y el poder de la ciencia y su autoridad, preocupado de construir teorías pertinentes para interpretar la modernidad, el desarrollo industrial y sus efectos, la cultura y la vida urbana. Hemos estado durante muchas décadas con la dificultad de articular dos dimensiones de una misma identidad: la producción de conocimiento científico y la construcción de una sociedad habitable para todos. Dos campos de actividad y dos caras de una misma disciplina se han tensionado. El de la universidad (los académicos) y los de la multiplicidad de oportunidades del campo no universitario. Pero la tendencia de las dos últimas décadas, ha ido acrecentando la valoración de la sociología aplicada y de intervención, buscando todos (académicos y profesionales), nuevas formas de profesionalización ante demandas del sector empresarial, de la educación y la capacitación, del sector público a nivel regional o local. Sin embargo, las diferencias no están resueltas.

Después de más de treinta años de construcción de un « oficio » de sociólogo centrado en la producción de un saber científico en el marco de un Estado-providencia, la crisis económica de los años 80, sufrida a veces con crudeza, no sólo por los pobres, sino también por las clases medias, las instituciones de base, cercanas a la gente, comenzaron a requerir profesionales que además de su capacidad de comprensión de los problemas sociales, sean capaces de ofrecer respuestas eficaces que solucionen situaciones concretas. Se ha acrecentado la demanda por profesionales que sepan de desarrollo urbano y de animación sociocultural, de prevención de delincuencia y de inserción de los excluidos, de los problemas de convivencia en los barrios y de conflictos familiares. En el contexto de las organizaciones productivas y de servicios se requieren profesionales capaces de implementar procesos

24 Desde 1970 existía l'Ecole Pratique de Recherche et Action en Sciences Sociales, (EPRAS) en l'EHESS. Y en 1975, los sociólogos mencionados crean en 1975 el ADSSA: Association pour le Developpement des Sciences Sociales Appliquées. 


\section{LA SOCIOLOGÍA PROFESIONAL Y SU LEGÍTIMA IDENTIDAD EN EL CAMPO DE LAS CIENCIAS SOCIALES / Gómez de Benito}

de modernización y rediseño de estructuras y procesos, además de la actualización de las relaciones sindicales.

Nuevo contexto, nuevas demandas y nuevas competencias operativas que se deben incluir en el nuevo oficio de sociólogo, más plural y flexible que el planteado por Bourdieu ${ }^{25}$. Estas nuevas formas prácticas de acción profesional de los sociólogos se han ido situando en un eje de saber hacer, y se expresan en formas de intervención o de evaluación sociológica en campos muy precisos y por clientes institucionales que tienen más claros los problemas que las soluciones.

Estas nuevas formas de intervención han ido exigiendo a los nuevos sociólogos, no sólo encuestar, sino ubicarse en el terreno mismo de los hechos. Se les está pidiendo que actúen interviniendo sobre los actores y sus culturas, a través de dispositivos de análisis y de apertura a otros actores. Se les está pidiendo también capacidad de innovación y creatividad, hacer uso no sólo de la razón, sino también de la imaginación y los sentimientos. Se está requiriendo saber acompañar procesos de aprendizaje de nuevas capacidades de acción, de representaciones diferentes de la realidad social, saber aprovechar la fuerza de aquellos que se interesan por cambiar, etc. Todo ello va a permitir comprender y actuar en las instituciones, los sistemas de actores y sus dinámicas de cambio y aprovechar las situaciones y las personas facilitando su rol profesional.

También hoy en un contexto de globalización con predominio neoliberal los sociólogos están siendo solicitados para explorar las consecuencias sociales y culturales de determinadas políticas y reformas en el sector público o en el privado. Saber observar resultados y medir impactos, saber comparar datos de resultados de situaciones diferentes o anteriores, construir bancos de datos etc. siguen siendo tareas propias del saber sociológico. 


\section{La pluralidad del campo profesional de la sociología en Chile ${ }^{26}$ :}

\section{Debates y tensiones actuales}

Si bien se ha considerado necesario contextualizar este proceso en la trayectoria de las transformaciones que experimentan las ciencias sociales, la recomposición de sus identidades, del campo de trabajo, de las nuevas prácticas y competencias de intervención de sus profesionales en otros contextos, este proceso de transformación que experimentan las prácticas y las identidades profesionales de las ciencias sociales en el marco de las profundas mutaciones sociales y culturales de las sociedades actuales, está estrechamente vinculado con el proceso de redefinición neoliberal del Estado y de sus relaciones con la sociedad civil.

Estamos hoy en presencia de una nueva fase de la historia de la Sociología. Por una parte, la masificación de la enseñanza universitaria de la sociología, durante los últimos cuarenta años, y por otra la diversificación de prácticas especializadas como demanda de servicios, tanto del sector público como del privado, hace que la práctica de la sociología se proyecte fuera de la Universidad, la experiencia francesa y el caso chileno así lo muestran.

Hoy la sociología, en medio de las transformaciones que experimenta lo social y la comprensión de lo social, continúa interrogándose sobre las dinámicas y tensiones identitarias que le atraviesan. Sin embargo, nuevos elementos se incluyen en la reflexión. Nuestro interés es de encontrar cuáles son las nuevas demandas de la sociedad actual sobre la sociología, como saber disciplinar y como saber hacer profesional. Estos nuevos requerimientos van conformando nuevas formas de ejercicio del oficio de sociólogo y de sus aplicaciones prácticas. Es esta la razón de la necesidad de una búsqueda sobre las nuevas formas que construyen hoy la identidad de los sociólogos, especialmente para quienes estamos en la tarea de formación de jóvenes profesionales de las ciencias sociales.

26 GOMEZ, J. y SANDOVAL M.: « Proyecto de Licenciatura en Sociología »; Universidad Católica Raúl Silva Henríquez, (Documento interno) Santiago, 2000. 


\section{LA SOCIOLOGÍA PROFESIONAL Y SU LEGÍTIMA IDENTIDAD EN EL CAMPO DE LAS CIENCIAS SOCIALES / Gómez de Benito}

Los cambios que afectan a los modelos político-económicos y su efecto en las instituciones sociales se han convertido en el principal factor de emergencia de demandas sociales y de desarrollo de nuevas prácticas profesionales de los sociólogos. Estas nuevas demandas no pueden satisfacerse desde las antiguas posiciones de un saber teórico o disciplinar. Los nuevos espacios se están abriendo en el campo empresarial, en la administración pública y privada, y las nuevas caras de la profesionalización de la sociología se han ido construyendo desde tareas tales como: consultores, formadores, administradores de recursos humanos, animadores culturales, educadores populares, planificadores, responsables de proyectos de acción social. ${ }^{27}$

Al desarrollo plural de las sociologías extrauniversitarias en Europa corresponde una pluralidad de espacios de ejercicio profesional de la sociología. En Francia, Bélgica, Alemania, en prácticamente toda la Unión Europea los sociólogos ejercen en campos extremadamente variados, que van desde la empresa clásica y alternativa a los colegios, las comunidades locales, la familia, el desarrollo rural, la ciudad, las instituciones de salud, la inserción y las diversas colectividades territoriales. Sin embargo, esta pluralidad conlleva tensiones y peligros de dispersión.

Una tensión permanece en la comprensión y el ejercicio de la sociología: una de naturaleza aplicada, orientada hacia las demandas del cliente. La otra de naturaleza científica, respondiendo a esquemas paradigmáticos de las ciencias sociales y a políticas de desarrollo académico y de la investigación en las Universidades. Para muchos sociólogos esta división sigue estando presente. Son dos mundos que no siempre se encuentran, en función de competencias, capitales, competencias específicas para responder a las demandas tan diferentes de cada campo.

De los sociólogos académicos se espera siempre sus corpus de conocimiento para definir teorías que permitan interpretar los grandes temas que preocupan a la sociedad moderna y a su gente. Un gran aporte de la construcción teórica de la sociología ha sido la fundamentación de la construcción y de las transformaciones sociales de la modernidad a través de la comprensión de los propios actores, de los sistemas organizados, de los movimientos sociales, de la relación entre los individuos y sus aportes

27 Este tema fue debatido en el SEMINARIO: "Nuevas identidades profesionales y nuevos campos para las ciencias sociales" realizado en nuestra Universidad entre el 10 y el 13 de Septiembre de 2001 
a la acción colectiva, etc.

En otro espacio los consultores analizan las disfunciones organizacionales, han descrito tipologías de funcionarios y autoridades, han evaluado los efectos de cantidad enorme de proyectos y reformas sociales, han acompañado procesos de cambio social, cultural y tecnológico. Los estudios aplicados responden a peticiones de sondeos para que en el sector público o privado los altos mandos puedan decidir. Con el soporte del saber recibido en la Universidad, los profesionales desarrollan bancos de datos y efectúan prácticas de intervención que no se preocupan o no les interesa ni explicitar, ni comunicar, porque el saber se va situando cada vez más en la esfera de lo privado. Las razones comerciales también se hacen presentes.

En nuestra realidad chilena, considerando los ejes dicotómico y transversales entre los que se sitúa la comprensión del ejercicio de la disciplina y las prácticas del sociólogo, en los espacios público y privado, es posible construir una tipificación de las prácticas sociológicas a partir de lo que Gabriel Salazar denomina «paradigmas fragmentarios» como parte de la «pulverización del saber»

\begin{tabular}{|c|c|c|}
\hline & CAMPO I & $\mathrm{ACCION}$ \\
\hline & PUBLICO & PRIVADO \\
\hline $\begin{array}{l}\text { PERSPECTIVA } \\
\text { DISCIPLINAR }\end{array}$ & $\begin{array}{c}\text { Tipo } 1 \\
\text { SOCIOLOGO } \\
\text { INTELECTUAL } \\
\text { Ubicación: UNIVERSIDAD } \\
\text { Tarea: Investigación-Docencia }\end{array}$ & \begin{tabular}{l}
\multicolumn{1}{c}{ Tipo 2} \\
\multicolumn{1}{c}{ SOCIOLOGO DE LA } \\
ACCION \\
Ubicación: Organizaciones \\
De base \\
Tarea: producir conocimiento \\
Con los actores y procesos \\
Socio-culturales
\end{tabular} \\
\hline$\frac{\mathrm{Y}}{\text { ppOFESIONA }}$ & $\begin{array}{c}\text { Tipo } 3 \\
\text { SOCIOLOGO POLITICo }\end{array}$ & $\begin{array}{c}\text { Tipo } 4 \\
\text { SOCIOLOGO CONSULTOR }\end{array}$ \\
\hline & $\begin{array}{l}\text { Ubicación : Esferas de } \\
\text { gobierno y adm.pública } \\
\text { directivo/tecnopolítica, } \\
\text { política pública y política } \\
\text { social } \\
\text { Tarea: Cargos públicos: } \\
\text { Asesoría y gestión en } \\
\text { Políticas públicas } \\
\text { Intervenciones/Estrategias }\end{array}$ & $\begin{array}{l}\text { Tarea: Estudios e } \\
\text { Intervenciones orientadas a la } \\
\text { toma de decisiones }\end{array}$ \\
\hline
\end{tabular}




\section{LA SOCIOLOGÍA PROFESIONAL Y SU LEGÍTIMA IDENTIDAD EN EL CAMPO DE LAS CIENCIAS SOCIALES / Gómez de Benito}

\section{1er. tipo: El SOCIOLOGO INTELECTUAL}

En lo que se refiere al sociólogo como intelectual y como político, en muchas partes del mundo, la génesis del quehacer sociológico se radica en la Universidad y los partidos políticos. Como se dijo anteriormente, Weber desarrolla profusamente el tema en «El político y el científico», vaticinando la compleja relación que habría de entablarse entre ambos mundos y que atraviesa la producción sociológica durante décadas. En el caso chileno esta realidad caracteriza la sociología de los años 50 - 70.

La experiencia indica que la formación de sociólogos en Chile, iniciada a finales de la década del 50, se orientó al cambio social: hacia la filosofía social-política (la orientación del cambio) y hacia el diagnóstico e intervención de las transformaciones estructurales de la sociedad chilena (como evaluación y prospectiva de los cambios acaecidos). Esta orientación disciplinar que buscó generar conocimientos en «Temas de actualidad» llevó a una prescindencia del sociólogo en el mercado laboral privado, en la medida que dichos conocimientos respondían a inquietudes futuristas, ideológicas (o al menos sospechosas de algún tipo de conspiración al interior del sistema) o, lo que es peor, carentes de significado desde el punto de vista de los requerimientos estructurantes de la sociedad en ese momento. Era una ciencia que incomodaba, en términos de Bourdieu.

En ese sentido, cabría hablar de la marginación (o automarginación, con frecuencia) de la vida organizacional típica de la sociedad moderna, la empresa, y a un enclaustramiento en organizaciones productoras de sentido y/o discurso, tales como las Universidades, centros autónomos de investigación y los partidos políticos. Esta práctica intelectual de la sociología se sitúa hoy en alguna de las grandes universidades del país. Pero también la presencia intelectual del sociólogo se hace notar desde pequeños espacios provenientes de algunas universidades privadas de pocos años de vida.

El auge de las Universidades privadas ha hecho que exista una cantidad también considerable de sociólogos dedicados a la práctica docente, a la investigación y gestión en la Educación Superior. Aunque no son 
muchos los que tienen una dedicación exclusiva, el mundo académico es un espacio laboral abierto lleno de posibilidades para el ejercicio de la docencia, la reflexión, el análisis, la investigación teórica o aplicada, los servicios profesionales al Estado o al sector privado. Las Universidades están siendo espacios con amplias posibilidades laborales para los sociólogos, especialmente por las vinculaciones y la legitimidad que éstas tienen entre la comunidad nacional.

\section{$2^{\circ}$ tipo: EL SOCIOLOGO DE LA ACCION}

A mediados de los años ochenta, la concepción del trabajo sociológico siguió siendo el de realizar estudios; aunque también existía la visión más militante, que consideraba que, además de interpretar la realidad es inherente a la sociología contribuir a un «nuevo orden», que haga la convivencia más justa y humana. En dicha situación, los sociólogos junto a los trabajadores sociales dedicaron su saber práctico a formar parte o a liderar procesos de intervención social (promoción humana, capacitación, organización, etc.) incluyendo la contribución de un saber sobre lo social a través de modalidades de investigación-acción.

El sociólogo de la acción se vuelca hacia la problemática social desde el punto de vista de la base social, está presente en organizaciones sociales y colaborando con los actores protagonistas y conductores de movimientos sociales. Durante las últimas décadas tuvieron que experimentar la tensión entre las dificultades de la represión y las dificultades del diálogo con el mundo académico, debido al aislamiento entre ellos y la vida universitaria.

Es posible concluir que la sociedad chilena, en un proceso de desarrollo acelerado, requiere que los sociólogos intervengan activamente en la elaboración y diseño, implementación, ejecución y evaluación de proyectos orientados a ese desarrollo. Es una demanda de la sociedad. Ya no es posible pensar que los sociólogos son un grupo privilegiado que mira la realidad desde las alturas de la criticidad absoluta y que sólo se comprometen con la realidad desde una óptica holística. La sociedad demanda una profesionalización creciente de la actividad sociológica, lo cual no quiere decir que lo otro (lo disciplinario) quede excluido, muy 


\section{LA SOCIOLOGÍA PROFESIONAL Y SU LEGÍTIMA IDENTIDAD EN EL CAMPO DE LAS CIENCIAS SOCIALES / Gómez de Benito}

por el contrario, recibirá más demanda. Lejos de funcionalizarse con el status quo, el perfil más genuino del sociólogo es el de interpretación y asesoría de los procesos de cambio, haciéndose presente como intelectual o como profesional en las instituciones sociales, sin renunciar, pero sin imponer la creencia decimonónica de que nuestra responsabilidad consiste en cambiar la sociedad.

\section{3er tipo: EI SOCIOLOGO POLITICO}

El sociólogo profesional al servicio del Estado (burócrata en su más genuino sentido sociológico) ha demostrado históricamente que el sociólogo es organizacionalmente útil y que el campo de la gestión de las políticas públicas desde los diferentes niveles de las instituciones del Estado ha sido también un espacio profesional legítimamente ganado y aprovechado por los sociólogos-políticos. Concretamente, las transformaciones relevantes en el plano educacional y de la salud, están incluyendo la contratación de sociólogos con grados de especialización en tales áreas.

A nivel del Estado, el tema de la modernización del sector público, la contingencia de la «modernización del Estado», ha ampliado las oportunidades profesionales para el sociólogo, como consecuencia de la profesionalización de un sinnúmero de tareas anteriormente colonizadas por prácticas tradicionales. De hecho, una cantidad importante de sociólogos han emigrado desde el mundo de las ONG's al mundo del Estado. Es decir, si aceptamos que existe un proceso de desarrollo económico inédito, ese proceso estará asociado a una modernización social, que amerita una reflexión y una práctica sociológica también inédita. Así pues, en relación con las oportunidades laborales, podemos constatar que el mercado laboral habitual de los sociólogos en los últimos 20 años ha sido el Estado, las Universidades y el mundo de las organizaciones no-gubernamentales ( $\left.\mathrm{ONG}^{\prime} \mathrm{s}\right)$, este último en franco retroceso.

La sociología debería ser capaz de articular y coordinar sus competencias críticas sin dejar de participar en aquello que la sociedad le demanda: el ejercicio pluridisciplinar y de complementación técnica con otras 
disciplinas (en especial, la economía, la administración, la educación y las disciplinas del ámbito de la salud); y mayores competencias técnicas que le permitan acceder meritocráticamente a los niveles decisionales del aparato estatal.

\section{$4^{\circ}$ Tipo: SOCIOLOGO CONSULTOR}

El ejercicio profesional del sociólogo consultor está condicionado por las demandas de los clientes. Estos pueden ser tanto del sector público como privado. Se trata de una sociología cuya contribución a la ciencia es un conocimiento práctico, desde y para la práctica. Se trata de un modo de ejercer la profesión que aunque no busca verdades respecto a las grandes interrogantes de la sociedad y la convivencia, aporta luz sobre problemas puntuales, de la sociedad o de las instituciones. Este paradigma no ofrece necesariamente una contribución a la acumulación del conocimiento, a menos que el profesional consultor lo haga en forma personal.

En el último tiempo, con modestia, pero crecientemente los sociólogos han logrado insertarse exitosamente en empresas privadas, preferentemente en el área de recursos humanos y el marketing, iniciándose ya un proceso de legitimación del oficio en ese campo laboral, produciéndose una creciente demanda por titulados de esta disciplina, los que, por diversos motivos, son escasos. Esta demanda es marcadamente profesional, aunque con componentes intelectuales cuando se trata de aportar en los procesos de planificación estratégica o en los diseños organizacionales.

Lo que el sociólogo tiene que ser en una organización es lo que en el mundo de la técnica son los «creativos»: Así como éstos últimos logran innovar en la tecnología posicionándose fuera de ella, los sociólogos consultores tienen que ser personas orientadas a la innovación en la gestión situándose fuera de ella. Ello implica una permanente actitud de interpretación de las relaciones sociales en los más diversos contextos sistémicos que permitan generar modelos adaptados para ser aplicados a la realidad de las instituciones.

Para concluir, queremos recordar lo que hace ya veinte años escribía Jean Remy sobre los dilemas del sociólogo: vocación u oficio, entre el 


\section{LA SOCIOLOGÍA PROFESIONAL Y SU LEGÍTIMA IDENTIDAD EN EL CAMPO DE LAS CIENCIAS SOCIALES / Gómez de Benito}

conocimiento y la intervención. Se pregunta nuestro viejo profesor de Lovaina, si evocando a Max Weber, el sociólogo « debe implicarse en una ética de responsabilidad, preocupado primero de participar en la puesta en práctica de opciones sociales, o al contrario, debe situarse en una ética de la convicción donde lo esencial es el aporte a un plan de una visión del mundo a construir » y proponía una reorientación de la Sociología a condición de seguir formando parte de la nueva sociedad que se inventa ${ }^{28}$. « La apertura interdisciplinaria debería suceder a los enclaustramientos. Más allá de las vinculaciones entre disciplinas capaces de explicar, podría intentarse una relación más estrecha en función de la intervención. Ello supone « el arte » de sanar, de construir, de regular. La apertura interdisciplinaria es tan importante a nivel de la comprensión de la realidad como en el de la acción por el cambio.

El oficio de sociólogo ha estado durante mucho tiempo implicado en la constitución de una disciplina de sabios o intelectuales, de investigadores y profesores en contextos universitarios. Estamos en un momento nuevo en el que el oficio de sociólogo se encuentra con nuevas demandas sociales, diferentes y diversificadas, según los países, pero que desafían a la apertura y a la respuesta a las oportunidades, a trabajar junto a otros profesionales $^{29}$. Esta sociología profesional puede ser concebida como un sistema de acción colectiva, como una capacidad de actuar desde saberes, valores, prácticas y competencias singulares.

Así pues, en el futuro la práctica de una sociología extraunivesitaria no puede ser considerada como ilegítima o marginal. Esta tendencia a ejercer el oficio de sociólogo fuera de la Universidad es ya una realidad en todo el mundo ${ }^{30}$. Los sociólogos están en el sector público y en el privado, en los grandes y pequeños ministerios, en instituciones de nivel nacional y local, al servicio del Estado, al servicio de la empresa privada, grande o pequeña, y al servicio de grupos y movimientos sociales.

28 REMY J. « Les dilemmes du sociologue: vocation o metier? »; Revue de l'Institud de Sociologie, ULB, 1980 , p. $21-28$

29 CHENAL, O. GUERRITSEN, D. SAINSAULIEU, R., y otros: «L'exercise professionnel de la sociologie », CNRS-IRESCO, París, 1987

30 La Revista Sociologie et Sociétés, de Quebec, dedicó en 1988 un nª a este tema. Jean Renaud en su presentación titula el problema como « la sociología invisible o la sociología fuera de la Universidad ». Estos sociólogos forman el principal grupo de miembros de la Asociación canadiense de sociólogos y antropólogos de lengua francesa (Acslf), indica este autor. 
Ante esta pluralidad de prácticas se hace necesario profundizar y distinguir entre oficio, profesión y disciplina. Hacer estas diferencias nos exige precisar los límites del objeto, integrar las prácticas en un sentido compartido. Puede ser que la disciplina sea el elemento integrador, desde la formación. Es necesario recuperar los genuinos significados del término profesión ${ }^{31}$ que se refieren al sentido de pertenencia a una comunidad. En nuestro caso la profesión puede tomar cuerpo en torno a una disciplina, principal fuente de integración. Y la formación disciplinar es tarea de la primera formación en la Universidad. Sin embargo, en el plano de la formación hay que saber incluir también nuevos saberes especializados y competencias utilizables en las prácticas profesionales. La distancia entre Universidad y mercado de trabajo es imprescindible acortarla. Así se ha asumido en muchos planes de estudio, como es nuestro caso, donde directamente se aborda la sociología desde una perspectiva orientada al campo profesional. ${ }^{32}$

31 DUBAR, C et TRIPIER, P: « Sociologie des professions »; Armand Colin , 1998

32 Bajo la dirección de M. Hirschorn, en la Universidad de Toulouse se inició en 1980 una « Maestría en Ciencias y técnicas de sociología aplicada ». Los planes de formación de Sociología en la mayor parte de las Universidades españolas dan opción a la formación profesional. En Chile la constante que se encuentra en las Universidades es la formación fundamentada en los dos grandes pilares inseparables: la teoría y la metodología de investigación. Varían los campos y las problemáticas que se abordan en las sociologías especiales, pero hay acuerdo en los pilares teórico-metodológicos. 


\section{LA SOCIOLOGÍA PROFESIONAL Y SU LEGÍTIMA IDENTIDAD EN EL CAMPO DE LAS CIENCIAS SOCIALES / Gómez de Benito}

BIBLIOGRAFIA

- ACSALF: Colloque 1981: «l'Intervenction sociale », Edit. Coop. Albert Saint Martín, París, 1982

- ANSART, PIERRE: 《Les sociologies contemporaines », Seuil, París, 1990 “-

«Sociologies de la modernité »

- BAJOIT G. y FRANSSEN A.: «Les transformations des métiers du secteur non-marchand;

Université Catholique de Louvain Bèlgique. BAJOIT, G.:

« Pour (re)donner du sens à la vie sociale »

in Travailler le social, (Revue trimestrel), No. 17, 1996,

Editée par CARDJIN Publications, LLN, Belgique

- BLANC, MAURICE, J. REMY, et All. :

«Vie quotidienne et democratie; Pour une sociologie de la transaction sociale »; Harmattan, París, 1994

- BERTHELOT, JEAN MICHEL «L'Intelligentia du social »; París, 1990

« La construction de la sociologie », PUF, Paris, 1991

- BENSIMON D;: 《Le metier du sociologue », APS, París, 1980

- BLANC M.: «La recherche appliquée en sociologie et les demandes sociales », Revue de l'Institut de Sociologie, $N^{\circ} 1 / 2$, ULB, Bruxelles

- BOURDIEU, P., CHAMbOREDON, J.CL., PASSERON, J CL. :

« Le metier de sociologue », Mouton-Bordas, París, 1969

- CASTELL, R.:

«Les métamorphoses de la question sociale », Gallimard, París, 1995

- CIVARD A.:

«Les métiers de la sociologie »; L’Etudiant, París, 1993

- COHEN-HUTHER J. «Les roles professionnels du sociologue extrauniversitaire », 